\title{
THE EFFECT OF MANHOLE SHAPE AND WALL THICKNESS ON STRESS STATE IN A CYLINDRICAL PRESSURE VESSEL
}

\author{
JERZY LEWIŃSKI \\ Poznan University of Technology, Institute of Applied Mechanics, Poland \\ e-mail: jerzy.lewinski@put.poznan.pl
}

\begin{abstract}
The paper describes attempts to find advantageous shape and configuration of a manhole located in the upper part of a cylindrical pressure vessel. Manholes of circular and elliptical cross sections have been considered. The manhole is placed vertically and symmetrically with respect to the cylindrical vessel. Three variants of the manhole walls have been taken into account. In the first variant, the manhole wall reaches only the cylinder surface. The manhole walls in two other variants are elongated inside the cylindrical vessel to various lengths. In the case of elliptical manholes two other variants were been considered, i.e. with their major axes situated in parallel or perpendicularly to the axis of symmetry of the cylindrical vessel. Results of the numerical calculation are presented in figures and tables.
\end{abstract}

Keywords: pressure vessel, manhole, manhole-vessel strength

\section{Introduction}

The problems related to pressure vessels, as being very important for safety and reliability of these structures, are extensively considered and investigated by many authors. Many works are devoted to optimal shape of the vessel. Special attention is paid to the junction between the vessel and its head since this region is usually subject to considerable stress concentration due to the edge effect arising there. This aspect was considered by Ventsel and Krauthammer (2001) who delivered a monograph presenting the strength and stability problems of plates and shells with the edge effect of cylindrical shells. In the paper by Błachut and Magnucki (2008), a review of strength, static stability and structural optimization of horizontal pressure vessels is presented.

Magnucki and Lewiński (2000) described the stress state arising in an untypical torispherical head composed of circular and polynomial parts. Magnucki and Lewiński (2003) presented optimal design of the ellipsoidal head with consideration of various thicknesses of the shell. Magnucki et al. (2004) presented optimization of ground-based cylindrical tanks with consideration their strength and stability. Lewiński and Magnucki (2010) considered a dished head of a pressure vessel subject to internal uniform pressure. They used a trigonometric series in order to find proper shape of the profile. A similar approach to the shape of a dished head of the pressure vessel was presented by the same authors (Lewiński and Magnucki, 2012). In order to design the required profile of the head they used Bézier curves. Further development of the method was demonstrated by Krużelecki and Proszowski (2012). They considered a larger range of shapes of a vessel head approximated by convex Bézier polynomials or by functions with free parameters. The optimal solutions were obtained with the use of the simulated annealing algorithm. Magnucki et al. (2002) solved the problem of stress minimization of a vessel with an ellipsoidal head.

Other regions of stress concentration occur in the vicinity of holes that must be placed in the vessel shell. Such holes are designed for placing nozzles or manholes in the vessel. According 
to specific purposes, the holes may be located in the cylindrical part of the vessel or in its head. These problems were considered by Carbonari et al. (2011) who presented integrated optimization of the shape of an axisymmetric vessel provided with a nozzle. They minimized the Huber-Mises mechanical stress in the whole vessel and, particularly, in the nozzle/vessel junction. Giglio (2003) compared two different methods of designing vessel nozzles, included in the ASME and VCR 1995 standards. He focused on the fatigue analysis of various pressure vessel nozzles. Liu et al. (2001) sought the optimal shapes of intersecting pressure vessels. They considered optimal profile of variable thickness nozzle connecting a spherical shell pressure vessel to a cylindrical nozzle. They have shown that the design with a protruding nozzle gives a better stress distribution than a flush nozzle.

The problems of proper quality of the manhole/vessel junction was considered by Frum (1995a,b). He described attempts aimed at avoiding circumferential cracking of the reactor vessel wall initiated in the vicinity of the manhole neck. Firstly, he measured temperature distribution at the reactor jacket. Afterwards, he developed a program including four basic steps: reduction of thermal stresses, elimination of discontinuities, improvement of material properties and improvement of the structure itself. Such an approach appeared to be very effective. Lianghai et al. (1996) investigated the effect of thermal overload on strength of welded structures. Their research pertained mainly to pressure vessels and the fittings that were welded to the vessels, inclusive of manholes. They found that the thermal overload is an effective method for reducing residual stress and the risk of vessel failure. Rao et al. (1997) discussed the reasons of failures of stainless steel pressure vessels and components welded to them. They focused chiefly on improper care during fabrication, welding, testing, and storage of the vessels, and mentioned such factors as residual stress, sensitization, iron contamination and saline atmosphere that worsened their strength. Bolton et al. (2002) considered the strength of Magnox reactor pressure vessel and the piping welded to it under condition of irradiation. They investigated mainly the welding technology used during the manufacturing process of the vessel. Borla (2002) presented the results of investigation of the effects of thermal fatigue, vibration and hydrogen attack on material and welds degradation in reactors. He considered possible repairs of the vessel and focused on appropriate production, service and repair procedures.

The present paper considers a cylindrical pressure vessel equipped with a manhole. The manhole is located vertically and symmetrically with respect to the vessel. In search of the most advantageous shape and configuration of the manhole, many variants divided into separate sets have been analyzed. It is assumed that the material of the manhole/vessel junction is of the same quality as the one of the whole vessel. It means that the welds should be perfectly laid and smoothed.

\section{Formulation of the problem}

Vessels are usually provided with holes that may be located in various positions. The present paper considers the strength of a pressure vessel subject to internal uniform pressure and equipped with a manhole vertically penetrating the upper part of the cylindrical shell of the vessel. The manhole axis intersects the axis of symmetry of the vessel. Only a dimensionless profile of the vessel is considered with radius of the cylindrical part of the vessel equal to $r=1$. Dimensionless thickness of the shell in the examples calculated here amounts to $t=0.02$. In the case of a cylindrical vessel without any holes or nozzles, the equivalent dimensionless stress generated in the shell by internal pressure is equal to $\widetilde{\sigma}_{e q}=\sqrt{3} / 2=0.866$, according to Lewiński and Magnucki (2010) . It is obvious that a manhole significantly disturbs the stress pattern in the vessel shell. Hence, the present paper is aimed at finding such a shape and configuration of the manhole as to minimize the stress concentration arising in the shell. 
Let us assume that the manhole is of circular cross section with its dimensionless diameter equal to $D=2 r_{m}=1$. Its cover is placed at dimensionless height above the upper generatrix of the vessel being equal to $h=0.24$. (Fig. 1a). It is also assumed that the manhole is located far enough from the vessel heads and, in consequence, the stress concentration arising in the head/cylinder junction does not collide with the one resulting from the manhole. This allows one to consider the case as a cylinder of infinite length with the manhole located anywhere in it. Such a system is distinguished by the vertical plane of symmetry that allows taking into account only its one-fourth part with a certain length of the cylindrical shell. Such an approach requires imposing proper boundary conditions on the model edges. The upper and bottom edges of the vessel, as laying at the vertical lengthwise plane of symmetry, must not displace in the lateral direction, i.e. $u_{x}=0$. On the other hand, the cross-section of the vessel, which divides the manhole in half, should remain planar. It means that $u_{z}=0$ at this edge. The upper edge of the manhole remains circular and, therefore, $u_{x}=u_{z}=0$. Additionally, the bottom vessel edge should be restrained in the vertical direction, i.e. $u_{y}=0$. The boundary conditions are shown in Fig. 1a.

(a)

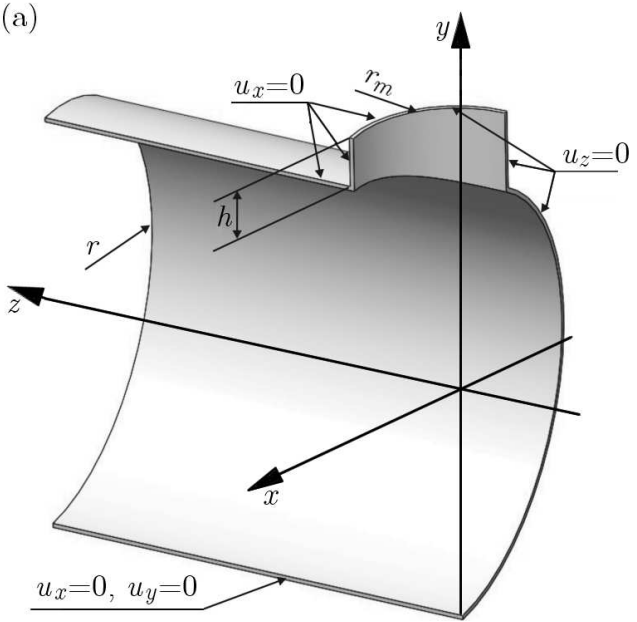

(b)

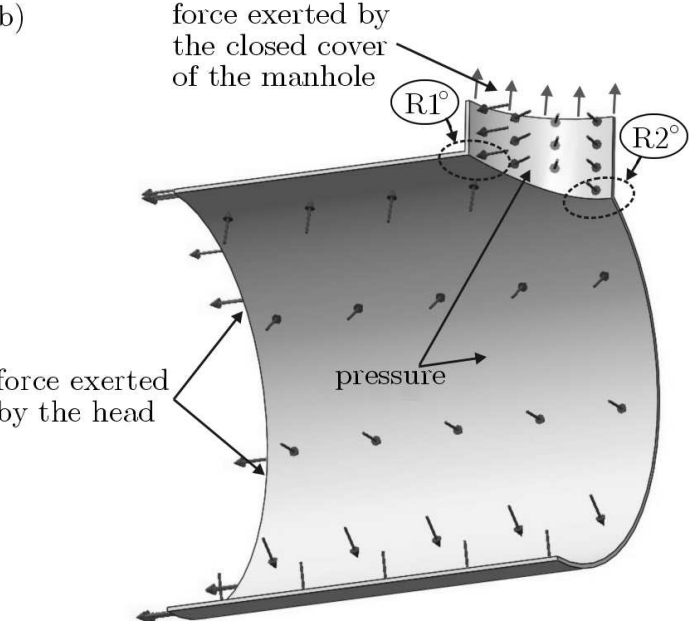

Fig. 1. Model of one-fourth part of the vessel with a manhole; (a) geometry, dimensions and boundary conditions marked at the edges; (b) the load: internal pressure and forces acting on the model boundaries, stress concentration regions $\mathrm{R} 1^{\circ}$ and $\mathrm{R} 2^{\circ}$

The model is loaded with internal pressure. Its value is so adjusted as to ensure easy comparison of the system to its dimensionless version, i.e. to ensure dimensionless membrane stress equal to $\widetilde{\sigma}_{e q}=0.866$ for the vessel without the manhole. The upper edge of the manhole is loaded with a vertical upward force equivalent to the pressure acting on the manhole cover. Similarly, the lateral edge of the vessel, located in the left hand side of the model, is loaded with a force caused by the vessel head, which is also subject to the pressure. The scheme of the load is shown in Fig. 1b. The regions $\mathrm{R} 1^{\circ}$ and $\mathrm{R} 2^{\circ}$ denoted in Fig. 1b indicate maximum stress concentrations arising in various variants of the manhole considered in the present paper.

All the manhole variants have been analyzed with the help of the finite element method by means of the educational version of the SolidWorks software. The meshes have been built of solid tetrahedral elements (with four Gauss points). An example of the mesh is shown in Fig. 2. Dimensions of the finite elements are significantly reduced in the region of manhole/vessel junction to improve the accuracy of computation. In all variants considered for the purposes of the present paper the meshes are similar, having about 650000 nodes and 450000 elements. 


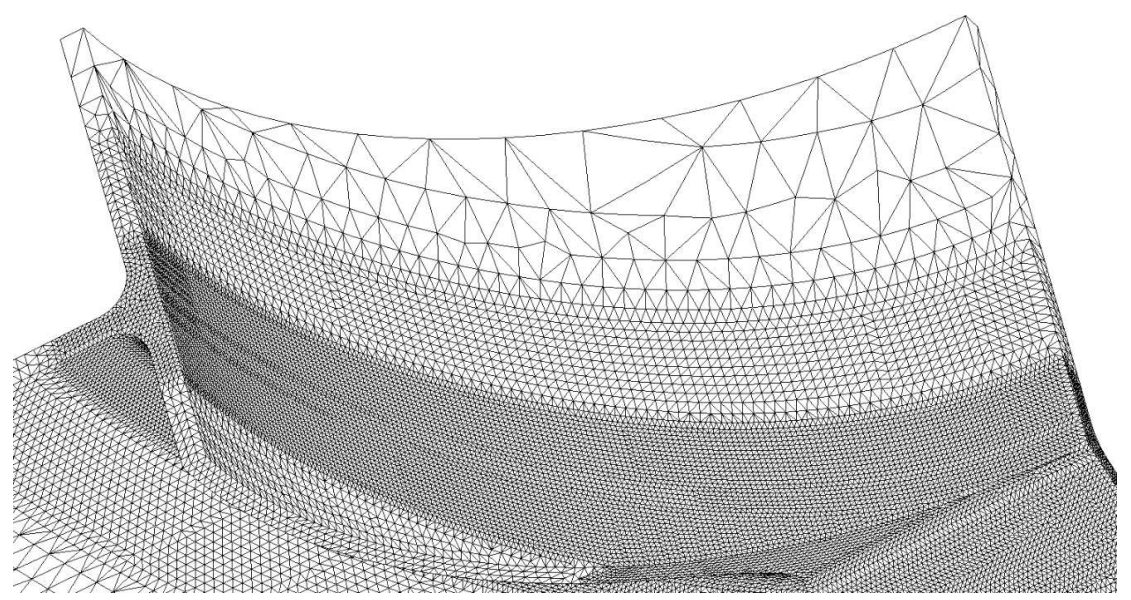

Fig. 2. Example of a part of the finite element mesh locally refined in the critical region

\section{Numerical results}

In the first basic variant, the side walls of the manhole reach exactly the vessel shell without penetrating it, as shown in Figs. 1a and 1b. The manhole wall thickness is equal to the one of the vessel. In this case, the maximum dimensionless equivalent stress amounts to 6.503 and occurs in the region $\mathrm{R} 1^{\circ}$ (Fig. 3 ).

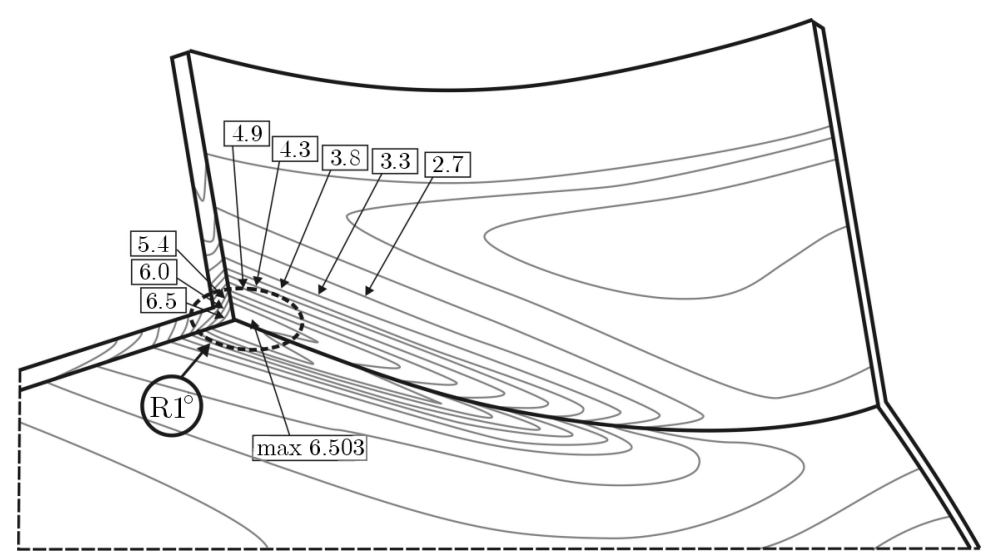

Fig. 3. Distribution of dimensionless equivalent stress in the region of manhole/vessel junction. The manhole reaches the vessel shell, manhole wall thickness equal to the one of the vessel

In order to improve the situation, the manhole wall has been elongated to a dimensionless length equal to $L=0.5$ (Fig. 4) so as to protrude from the inner surface inside the vessel for a moderate length. As a result, the manhole obtained form of a cylinder of length $L$ intersecting with the vessel. In this variant, the maximum dimensionless equivalent stress is equal to $\widetilde{\sigma}_{e q}=6.218$, approximately in the same place as before. Hence, it gives only insignificant improvement.

A better result has been obtained by rounding the sharp edges of the manhole/cylinder junction. Dimensionless radius of the edge rounding is equal to $\rho=0.03$, and finally the manhole/vessel region assumed form shown in Fig. 4. Such an approach allows reducing the maximum dimensionless stress down to $\widetilde{\sigma}_{e q}=4.43$, which occurs in the same location as before.

Further attempts to reduce the stress concentration consist in changing the manhole wall thickness, which is no longer equal to the one of the vessel. Dimensionless thickness of the manhole wall vary from $t_{m}=0.012$ to $t_{m}=0.060$ and the results obtained in this series of the variants are shown in Table 1. In the case of low thickness of the wall, changing from $t_{m}=0.012$ 


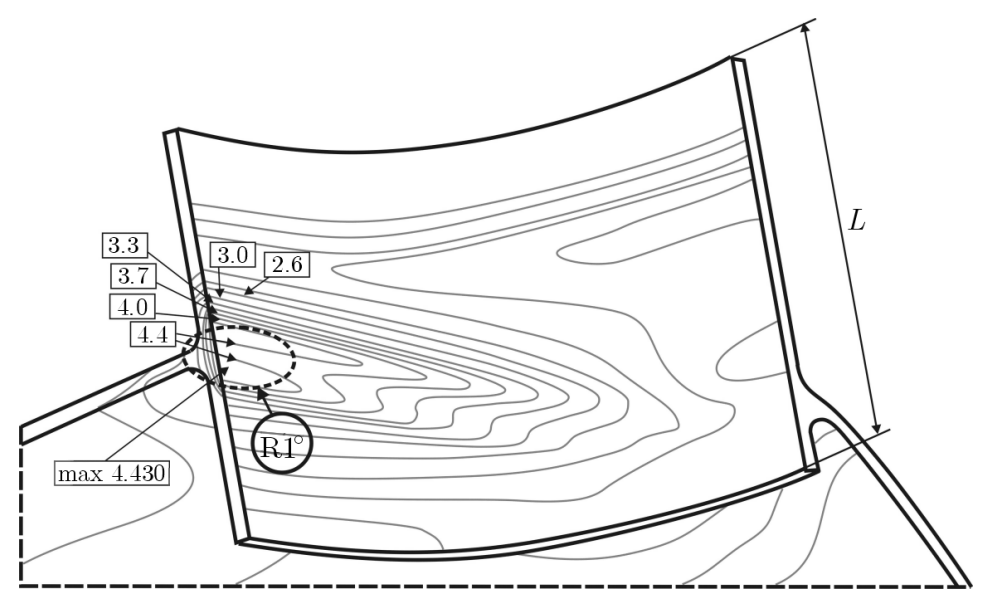

Fig. 4. Distribution the dimensionless equivalent stress in the region of manhole/vessel junction. The manhole embedded into the vessel, its wall thickness equal to that of the vessel

Table 1. Maximum dimensionless equivalent stress and location of stress concentration obtained for various values of manhole wall thickness

\begin{tabular}{|c|c|c|}
\hline $\begin{array}{l}\text { Manhole wall } \\
\text { thickness } \times 10^{-3}\end{array}$ & $\begin{array}{c}\text { Dimensionless } \\
\text { equivalent stress }\end{array}$ & $\begin{array}{c}\text { Location of stress } \\
\text { concentration }\end{array}$ \\
\hline 12 & 6.254 & \multirow{9}{*}{$\mathrm{R} 1^{\circ}$} \\
\hline 16 & 5.084 & \\
\hline 20 & 4.430 & \\
\hline 22 & 4.148 & \\
\hline 24 & 3.903 & \\
\hline 25 & 3.779 & \\
\hline 25.4 & 3.733 & \\
\hline 25.6 & 3.722 & \\
\hline 25.8 & 3.705 & \\
\hline 25.9 & 3.798 & \multirow{5}{*}{$\mathrm{R} 2^{\circ}$} \\
\hline 26 & 4.013 & \\
\hline 28 & 5.292 & \\
\hline 40 & 5.729 & \\
\hline 60 & 9.304 & \\
\hline
\end{tabular}

to $t_{m}=0.0258$, the maximum stress appears in the location marked as $\mathrm{R} 1^{\circ}$. The lowest value of the maximum stress corresponds just to dimensionless thickness equal to $t_{m}=0.0258$ and amounts to $\widetilde{\sigma}_{e q}=3.705$. Further growth of the thickness induces higher maximum stress but, at the same time, the location of stress concentration changes to the region R2 ${ }^{\circ}$ (Fig. 5).

The manhole having dimensionless length equal to $L=0.5$ penetrates the vessel, with $\rho=0.03$ rounding of sharp edges of the manhole/vessel junction.

Such a change of the maximum stress location is presumably caused by excessive stiffness of the junction between the manhole and vessel. Once the thickness exceeds the most advantageous value equal to $t_{m}=0.0258$, the stiffness grows together with increasing thickness of the manhole wall causing further growth of the stress.

A question arises whether the manhole must be of circular cross section. Hence, an alternative elliptical shape has also been checked with the unchanged surface area of the manhole cross section. Such a manhole shape ensures better comfort of the worker who enters inside the vessel. An ellipse having the semi-axes ratio equal to $5 / 4$ is assumed. As a result, dimensionless lengths of its semi-axes are equal to $a=0.559$ and $b=0.447$. Such a semi-axes ratio corresponds to 


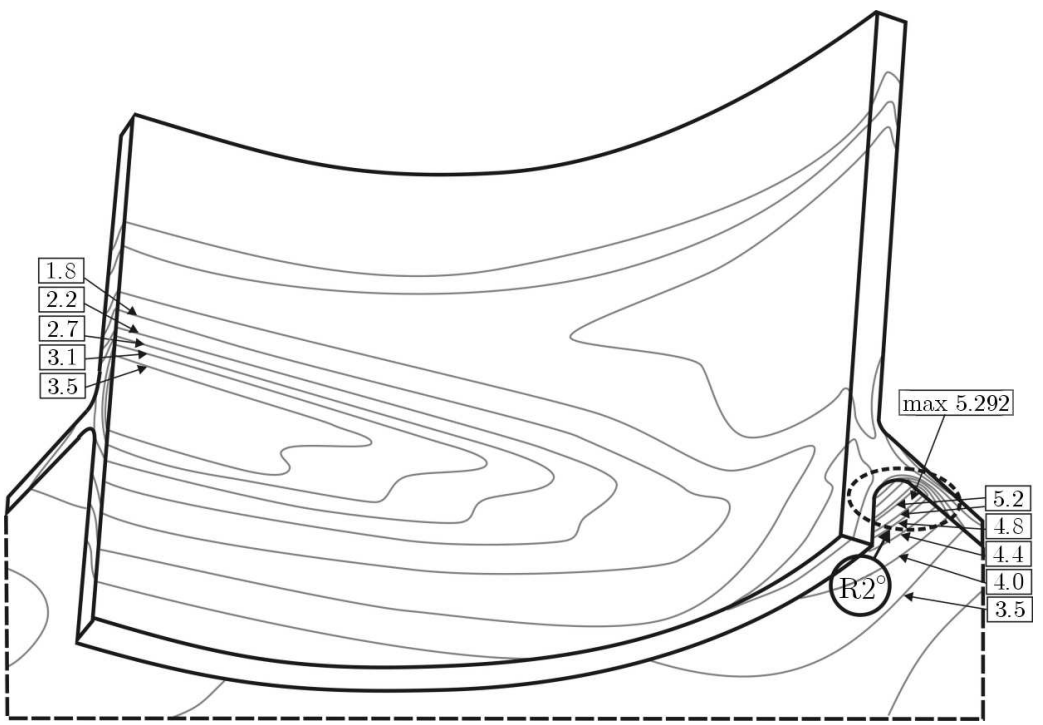

Fig. 5. Distribution of the dimensionless equivalent stress in the region of manhole/vessel junction. The manhole penetrates the vessel, its dimensionless wall thickness is equal to 0.028

an ellipse which does not much deviate from circular shape and, at the same time, the passage through it is quite comfortable.

For the elliptical manhole cross section, two basic orientations of its shape are possible: with the major semi-axis situated length-wise or cross-wise with regard to the vessel axis of symmetry. In the preliminary case, when the side walls of the elliptic manhole have thickness equal to thickness of the vessel and reach the vessel shell without penetrating it, the maximum dimensionless equivalent stress amounts to $\widetilde{\sigma}_{e q}=8.583$ for the length-wise and $\widetilde{\sigma}_{e q}=5.321$ for the cross-wise orientation of the ellipse major semi-axis. In both cases, it occurs in the location $\mathrm{R} 1^{\circ}$.

Comparison to the stress level equal to $\widetilde{\sigma}_{e q}=6.503$ which has been obtained in the similar case of the circular manhole shape allows one to presume that the cross-wise orientation is more advantageous.

Similarly as before, the manhole wall has been elongated to dimensionless length equal to $L=0.5$. As a result, the manhole obtained form of an elliptical cylinder penetrating the vessel. Without rounding the sharp edges arising in the manhole/vessel junction, the maximum dimensionless equivalent stress amounts this time to $\widetilde{\sigma}_{e q}=7.833$ for the length-wise and $\widetilde{\sigma}_{e q}=5.431$ for the cross-wise orientation of the ellipse major semi-axis. In both cases, it occurs again in the location $\mathrm{R} 1^{\circ}$. These two cases are presented in Table 2.

Table 2. Maximum dimensionless equivalent stress and location of maximum stress concentration obtained for elliptical cross-section of the manhole without rounding sharp edges of the manhole/vessel junction. Dimensionless manhole thickness is equal to $t_{m}=0.02$

\begin{tabular}{|l|c|c|c|}
\hline & $\begin{array}{c}\text { Length-wise } \\
\text { orientation } \\
\text { of major semi-axis }\end{array}$ & $\begin{array}{c}\text { Cross-wise } \\
\text { orientation } \\
\text { of major semi-axis }\end{array}$ & $\begin{array}{c}\text { Location } \\
\text { of stress } \\
\text { concentration }\end{array}$ \\
\hline \hline $\begin{array}{l}\text { Manhole wall reaches } \\
\text { vessel surface }\end{array}$ & 8.583 & 5.321 & \multirow{2}{*}{$\mathrm{R} 1^{\circ}$} \\
\cline { 1 - 3 } $\begin{array}{l}\text { Manhole wall elongated to } \\
\text { dimensionless length } L=0.5\end{array}$ & 7.833 & 5.431 & \\
\hline
\end{tabular}


The cross-wise orientation of the elliptic shape gives promising results, encouraging one to round the edges of the manhole/vessel junction as before, and to check what is the effect of variation of the manhole wall thickness. A series of such numerical experiments has been carried out. The results are shown in Table 3.

Table 3. Maximum dimensionless equivalent stress and location of maximum stress concentration obtained for elliptical cross-section of the manhole, with $\rho=0.03$ rounding of sharp edges of the manhole/vessel junction

\begin{tabular}{|c|c|c|c|}
\hline $\begin{array}{c}\text { Manhole wall } \\
\text { thickness } \times 10^{-3}\end{array}$ & $\begin{array}{c}\text { Length-wise orientation } \\
\text { of major semi-axis }\end{array}$ & $\begin{array}{c}\text { Cross-wise orientation } \\
\text { of major semi-axis }\end{array}$ & $\begin{array}{c}\text { Location of stress } \\
\text { concentration }\end{array}$ \\
\hline \hline 12 & 8.740 & 6.717 & \multirow{2}{*}{$\mathrm{R} 1^{\circ}, \mathrm{R} 2^{\circ}$} \\
\hline 14 & 7.959 & 7.417 & \\
\hline 16 & 7.218 & 8.046 & \multirow{2}{*}{$\mathrm{R} 2^{\circ}, \mathrm{R} 2^{\circ}$} \\
\hline 20 & 6.367 & 9.056 & \\
\hline 22 & 6.972 & 9.585 & \\
\hline 24 & 7.608 & 10.134 & \\
\hline
\end{tabular}

As opposed to the previous case of circular cross section of the manhole, the rounding worsens the situation. It is presumably caused by the fact that for the cross-wise orientation of the ellipse, the manhole reaches farther from the symmetry plane of the vessel and the rounded edges stiffen the junction more efficiently than in the case of the circular profile of the manhole. The region $\mathrm{R} 1^{\circ}$ is subject to the maximum stress only for the length-wise orientation of the major semi-axis and dimensionless thickness not exceeding $t_{m}=0.016$. In all other cases, the stress concentration moves to $\mathrm{R} 2^{\circ}$, and the stiffening effect becomes more evident with growing thickness of the manhole wall.

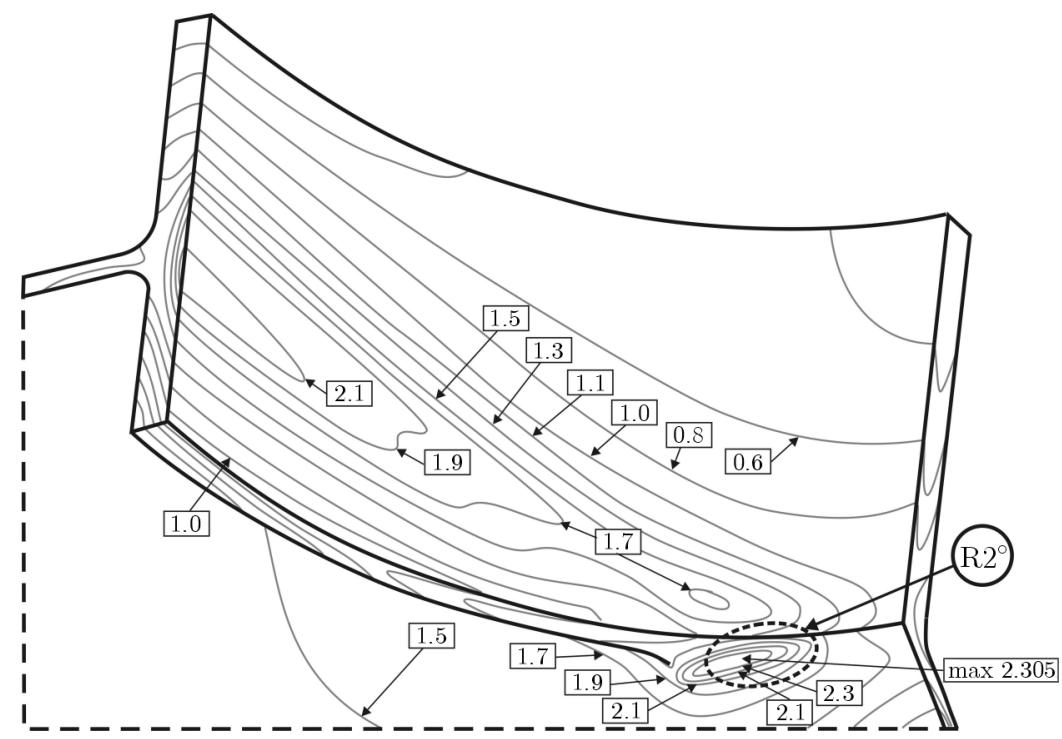

Fig. 6. Distribution of the dimensionless equivalent stress in the region of junction between the elliptical manhole and the vessel with cross-wise orientation of the ellipse major semi-axis. The manhole enbedded into the vessel and flushed with the inner wall of the vessel in the region $\mathrm{R} 2^{\circ}$, its dimensionless wall thickness equal to $t_{m}=0.036$

Excessive stiffness of the region $\mathrm{R} 2^{\circ}$ of the manhole/vessel junction suggests another solution of the manhole configuration. It seems that the manhole should be so elongated as to avoid too deep penetration into the vessel. The elliptical cylinder of the manhole should be flushed with the inner wall of the vessel in the region $\mathrm{R} 2^{\circ}$. Such a solution is shown in Fig. 6 . The stress 
concentration in it has been examined for a series of variants with dimensionless thickness of the manhole wall varying from $t_{m}=0.02$ to $t_{m}=0.05$. The maximum stresses and their locations are shown in Table 4.

Table 4. Maximum dimensionless equivalent stress and location of stress concentration obtained for various values of the manhole wall thickness. The elliptic manhole with semi-axes equal to $a=0.559$ and $b=0.447$ is elongated so as to be flushed with the inner wall of the vessel in the region $\mathrm{R} 2^{\circ}$. Sharp edges of the manhole/vessel junction are rounded with radius $\rho=0.03$

\begin{tabular}{|c|c|c|}
\hline $\begin{array}{c}\text { Manhole wall } \\
\text { thickness } \times 10^{-3}\end{array}$ & $\begin{array}{c}\text { Dimensionless } \\
\text { equivalent stress }\end{array}$ & $\begin{array}{c}\text { Location of stress } \\
\text { concentration }\end{array}$ \\
\hline \hline 18 & 3.765 & \multirow{3}{*}{ R $1^{\circ}$} \\
\hline 20 & 3.479 & \multirow{3}{*}{} \\
\hline 22 & 3.220 & \\
\hline 24 & 2.992 & \multirow{3}{*}{$\mathrm{R} 2^{\circ}$} \\
\hline 30 & 2.450 & \multirow{3}{*}{} \\
\hline 31 & 2.375 & \\
\hline 32 & 2.366 & \\
\hline 34 & 2.335 & \\
\hline 36 & 2.305 & \\
\hline 40 & 2.251 & \\
\hline 50 & 2.114 & \\
\hline
\end{tabular}

Among all the above considered manhole variants, this one is the most advantageous. With the growing wall thickness, the stress drops monotonically, only the region of maximum stress concentration moves from $\mathrm{R} 1^{\circ}$ to $\mathrm{R} 2^{\circ}$ for the thickness exceeding $t_{m}=0.031$. Such a result allows one to assume that the elliptical manhole profile with the cross-wise orientation of the ellipse and the elliptical cylinder of the manhole flushed with the inner wall of the vessel in the region $\mathrm{R} 2^{\circ}$ should be recommended as the best solution.

One might suppose that the manhole wall could be shorter also near the region $\mathrm{R} 1^{\circ}$. Figure 6 shows that the dimensionless equivalent stress in the bottom part of the manhole wall slightly drops below 1 in this location, approximating the level $\widetilde{\sigma}_{e q}=0.866$ existing in the vessel wall. Therefore, such length of the manhole may be considered as appropriate.

\section{Conclusions}

The proper shaping and location of a manhole situated in a pressure vessel appears to be very important from the point of view of the strength and reliability of the structure. In the case of a manhole fixed vertically and symmetrically in the upper wall of the pressure vessel there are some possible variants of its configuration.

- Manholes usually have circular cross section. In such a case, the manhole cylinder should be extended to a certain depth into the vessel and, at the same time, sharp edges of the manhole/vessel junction should be rounded. Depending on thickness of the manhole wall, the maximum stress concentration arises either in the region $\mathrm{R} 1^{\circ}$ or $\mathrm{R} 2^{\circ}$ (Fig. 1b). For the manhole thickness $t_{m}$ below the limit value equal about to 1.30 of the vessel thickness $\left(t_{m}<1.30 t_{v}\right)$, the stress concentration is located in $\mathrm{R} 1^{\circ}$. The lowest stress occurs for $t_{m}=1.30 t_{v}$ and it is the most advantageous solution of such a manhole. Once the manhole thickness exceeds this limit and increases, the stress concentration translocates to $\mathrm{R} 2^{\circ}$ and the stress level grows (Table 1). 
- A manhole of elliptical shape has been assumed as an alternative solution. In order to enable its comparison to the circular one, the manhole cross-section area has been maintained equal to the previous circular case. Finally, an ellipse having the semi-axes ratio equal to $5 / 4$ has been assumed. Such a manhole shape ensures better comfort of the worker who enters inside the vessel. The elliptical manhole may be pointed with its major semi-axis situated length-wise or cross-wise with regard to the vessel axis of symmetry. It appears that the cross-wise orientation is a better solution. Moreover, the elliptical cylinder of the manhole should be extended into the vessel so as to be flushed with the inner wall of the vessel in the region $\mathrm{R}^{\circ}$ (Fig. 6). In such a case, the maximum stress decreases with growing thickness of the manhole. Nevertheless, its excessive growth results only in an insignificant stress reduction. An examplary solution shown in Fig. 6, in which the manhole thickness is nearly twice as large as the one of the vessel $\left(t_{m}=1.80 t_{v}\right)$ seems to be sufficient.

It should be noticed that in the case of such a solution of the manhole/vessel junction, the maximum stress remains relatively high as compared to the reference level of dimensionless equivalent stress $\widetilde{\sigma}_{e q}=0.866$ existing in the vessel shell. Further improvement of the situation might be presumably achieved by more sophisticated shaping of the manhole/vessel junction, with special consideration of the region $\mathrm{R} 2^{\circ}$.

\section{References}

1. BŁachut J., Magnucki K., 2008, Strength, stability, and optimization of pressure vessels: Review of selected problems, Applied Mechanics Reviews, 61, 6, 1-33

2. Bolton C.J., Bischler P.J.E., Wootton M.R., Moskovic R., Morri J.R., Pegg H.C., Haines A.B., Smith R.F., Woodman R., 2002, Fracture toughness of weld metal samples removed from a decommissioned Magnox reactor pressure vessel, International Journal of Pressure Vessels and Piping, 79, 685-692

3. Borla J.Z., 2002, Parent material and weldments degradation on SASOL reduction reactors due to combined effect of thermal fatigue, vibration and hydrogen attack, International Journal of Pressure Vessels and Piping, 79, 577-584

4. Carbonari R.C., Munoz-Rojas P.A., Andrade E.Q., Paulino G.H., Nishimoto K., SoLVA E.C.N., 2011, Design of pressure vessels using shape optimization: An integrated approach, International Journal of Pressure Vessels and Piping, 88, 198-212

5. Frum Y., 1995a, Elimination of Circumferential Cracking in Reactor's Internal Jacket, International Journal of Pressure Vessels and Piping, 61, 383-409

6. Frum Y., 1995b, Time Related Temperature Field over a Reactor Internal Surface, International Journal of Pressure Vessels and Piping, 61, 367-381

7. Giglio M., 2003, Fatigue analysis of different types of pressure vessel nozzle, International Journal of Pressure Vessels and Piping, 80, 1-8

8. Krużelecki J., Proszowski R., 2012, Shape optimization of thin-walled pressure vessel end closures, Structural and Multidisciplinary Optimization, 46, 739-754

9. Lewiński J., Magnucki K., 2010, Shaping of a middle surface of a dished head of a circular cylindrical pressure vessel, Journal of Theoretical and Applied Mechanics, 48, 2, 297-307

10. Lewiński J., Magnucki K., 2012, Optimal shaping of middle surface of a dished head of circular cylindrical vessel with the help of Bézier curve, EngOpt 2012 - 3rd International Conference on Engineering Optimization, Rio de Janeiro, Brazil

11. Lianghai Z., Xuemong S., Hengyun J., 1996, Influence o warm overloading ion stress-corrosion cracking of welded structures, International Journal of Pressure Vessels and Piping, 69, 91-96 
12. Liu J.S, Parks G.T., Clarkson P.J., 2001, Shape optimization of axisymmetric cylindrical nozzles in spherical pressure vessels subject to stress constraints, International Journal of Pressure Vessels and Piping, 78, 1, 1-9

13. Magnucki K., LeWiński J., 2000, Fully stressed head of a pressure vessel, Thin-Walled Structures, 38, $167-178$

14. Magnucki K., Lewiński J., 2003, Optimal design of an ellipsoidal head of a pressure cylindrical vessel, Proceedings in Applied Mathematics and Mechanics, 3, 517-518

15. Magnucki K., Lewiński J., Stasiewicz P., 2004, Optimal sizes of a ground-based horizontal cylindrical tank under strength and stability constraints, International Journal of Pressure Vessels and Piping, 81, 227-233

16. Magnucki K., Szyc W., Lewiński J., 2002, Minimization of stress concentration factor in cylindrical pressure vessels with ellipsoidal heads, International Journal of Pressure Vessels and Piping, 79, 841-846

17. Rao B.S.C., Madeswaran R., Chandromohan R., 1997, In-fabrication and pre-service care on stainless steel pressure vessels, International Journal of Pressure Vessels and Piping, 73, 53-57

18. Ventsel E., Krauthammer T., 2001, Thin Plates and Shells. Theory, Analysis and Applications, Basel: Marcel Dekker, Inc, New York 